\title{
Tapered diode-pumped continuous-wave alexandrite laser
}

\author{
Ersen Beyatli, ${ }^{1}$ Ilyes Baali, ${ }^{2}$ Bernd Sumpf, ${ }^{3}$ Götz Erbert, ${ }^{3}$ Alfred Leitenstorfer, ${ }^{4}$ \\ Alphan Sennaroglu, ${ }^{1}$ and Umit Demirbas ${ }^{2,4, *}$ \\ ${ }^{1}$ Laser Research Laboratory, Departments of Physics and Electrical-Electronics Engineering, Koç University, \\ Rumelifeneri, Sariyer, Istanbul 34450, Turkey \\ ${ }^{2}$ Laser Technology Laboratory, Department of Electrical and Electronics Engineering, Antalya International University, \\ 07190 Dosemealti, Antalya, Turkey \\ ${ }^{3}$ Ferdinand-Braun-Institut, Leibniz Institut für Höchstfrequenztechnik, D-12489 Berlin, Germany \\ ${ }^{4}$ Department of Physics and Center for Applied Photonics, University of Konstanz, D-78464 Konstanz, Germany \\ *Corresponding author: umit79@alum.mit.edu
}

Received August 5, 2013; revised October 12, 2013; accepted October 14, 2013; posted October 15, 2013 (Doc. ID 195224); published November 12, 2013

\begin{abstract}
We describe a low-cost and efficient alexandrite $\left(\mathrm{Cr}: \mathrm{BeAl}_{2} \mathrm{O}_{4}\right)$ laser that is pumped by a high-brightness tapered diode laser (TDL). The tapered diode (TD) provides up to $1.1 \mathrm{~W}$ of output power and its wavelength can be finetuned to either $680.4 \mathrm{~nm}$ (R1 line) or $678.5 \mathrm{~nm}$ (R2 line) for efficient in-line pumping. Continuous-wave (cw) output powers of $200 \mathrm{~mW}$, slope efficiencies as high as $38 \%$, and a cw tuning range extending from 724 to $816 \mathrm{~nm}$ have been achieved. To the best of our knowledge, the cw power levels and slope efficiencies are the highest demonstrated so far from such a minimal complexity and low-cost system based on the alexandrite gain medium. Consequently, TDs operating in the red spectral region have the potential to become the standard pump sources for cw alexandrite lasers in the near future. (c) 2013 Optical Society of America
\end{abstract}

OCIS codes: (140.3580) Lasers, solid-state; (140.3480) Lasers, diode-pumped; (160.3380) Laser materials;

(140.3600) Lasers, tunable; (140.7300) Visible lasers.

http://dx.doi.org/10.1364/JOSAB.30.003184

\section{INTRODUCTION}

Optical gain media with broad emission bandwidths are highly versatile and enable extended wavelength tuning, as well as the generation and amplification of ultrashort optical pulses. If these active laser media also posses suitable absorption bands that allow direct diode pumping, this brings many additional advantages, such as lower cost [1, 2$]$, compactness [3,4], higher electrical-to-optical conversion efficiency [5,6], and lower noise [7]. Ti:sapphire has the broadest gain bandwidth among all the solid-state laser media, facilitating tuning from 680 to $1180 \mathrm{~nm}$ [8], as well as direct generation of pulses as short as $5 \mathrm{fs}$ [9]. The ideal pump wavelength for Ti:sapphire is around $500 \mathrm{~nm}$ [10]. However, due to the lack of suitable low-cost and high-power diode lasers in this spectral region, currently Ti:sapphire lasers are pumped by GaN diodes at $450 \mathrm{~nm}$ [11-14]. In a recent work, using two $1.2 \mathrm{~W}$ multimode single-emitter diodes as the pump source, Durfee et al. generated 15 fs pulses with $34 \mathrm{~mW}$ average power from a Kerr-lens mode-locked Ti:sapphire laser [13]. Continuous-wave (cw) powers as high as $159 \mathrm{~mW}$ and mode-locked powers as high as $101 \mathrm{~mW}$ (111 fs long pulses) have also been reported from a similar diode-pumped system [15]. Progress in directly diode-pumped Ti:sapphire laser systems is promising. Currently, the optical-to-optical conversion efficiency of the systems is rather low (5\%), partly due to the low pump beam quality and high passive losses of the Ti:sapphire gain medium [11]. We also note here that frequency-doubled-tapered diode lasers (TDLs) have also been recently demonstrated as a possible pump source for Ti:sapphire [16].

Similar to Ti:sapphire, chromium ${ }^{3+}$-doped laser materials also possess broad emission bands around $800 \mathrm{~nm}$. Moreover, their strong and broad absorption bands in the visible region of the spectrum enable wavelength and polarization flexible direct diode pumping by low-cost red diodes (630-690 nm). There exists a relatively large number of $\mathrm{Cr}^{3+}$-doped materials in which lasing has been reported [17]. Only a few, like Cr:colquiriites (Cr:LiSAF [18], Cr:LiCAF [17], Cr:LiSGaF [19]), emerald ( $\left.\mathrm{Cr}^{\mathrm{B}} \mathrm{B}_{3} \mathrm{Al}_{2}\left(\mathrm{SiO}_{3}\right)_{6}\right)$ [20,21], and alexandrite $\left(\overline{\mathrm{Cr}}: \mathrm{BeAl}_{2} \mathrm{O}_{4}\right.$ ) [22-26] are suitable for efficient laser operation with slope efficiencies above $50 \%$ since most of the other candidates suffer from strong excited-state absorption. In this work, our attention has been focused on alexandrite due to its blueshifted gain profile compared to Cr:colquiriites. The peak emission of alexandrite is around $750 \mathrm{~nm}$. This wavelength is quite important for optimum excitation of several widely used fluorophores in multiphoton microscopy, including 4',6-diamidino-2-phenylindole dihydrochloride (DAPI), Alexa flour 488, indo-1, and fura-2 [27-30].

Alexandrite (Cr-doped chrysoberyl) was first shown to lase in 1978 by Walling et al. [23]. It has many superior optical and thermal properties (see Table 1), including an intrinsic slope efficiency of $65 \%$ [36], a broad tuning range from 700 to $860 \mathrm{~nm}$ [24,33], lack of concentration quenching of fluorescence lifetime [31], and, interestingly, increased laser performance at elevated temperatures above room temperature [33]. Due to 
Table 1. Comparison of the Spectroscopic and Laser Parameters of the Gain Media Ti:sapphire, Cr:LiSAF, and Alexandrite

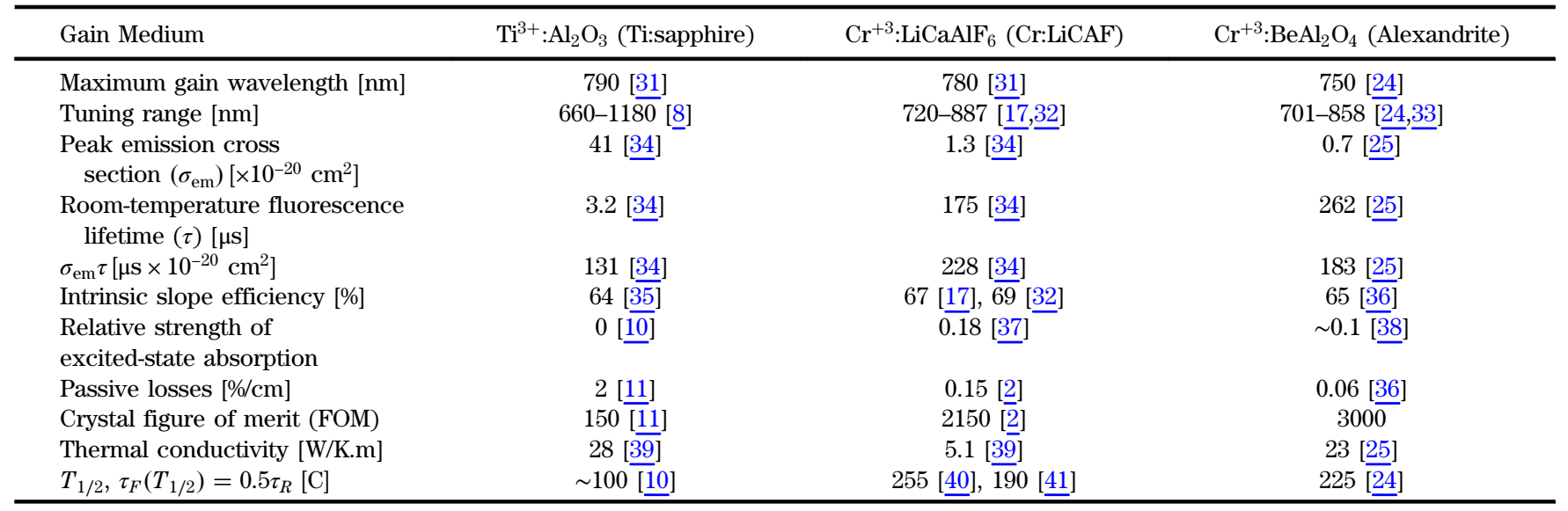

all of these, alexandrite was one of the most popular tunable lasers on the market during the 1980s [31]. This continued until the emergence of Ti:sapphire systems, at which time attention paid to alexandrite lasers began to diminish. However, we believe that with the recent progress in laser diode technology, alexandrite lasers can now offer a low-cost, efficient, and compact alternative to the existing Ti:sapphire laser technology in selected areas of applications, especially for multiphoton microscopy.

Figure 1 shows a simplified energy level structure for alexandrite in $E \| b$ orientation. Transitions from the ground state to the vibronically broadened ${ }^{4} T_{1}$ and ${ }^{4} T_{2}$ states generate two broad (FWHM around $100 \mathrm{~nm}$ ) and strong absorption bands with peaks around 410 and $590 \mathrm{~nm}$ [23]. These states have relatively short lifetimes (the intrinsic lifetime of the ${ }^{4} T_{2}$ state is around $6.6 \mu \mathrm{s})$. Moreover, similar to ruby [42], the metastable ${ }^{2} E$ state lies below the vibronically broadened ${ }^{4} T_{2}$ level in alexandrite. Hence, ions excited to the ${ }^{4} T_{1}$ and ${ }^{4} T_{2}$ states rapidly decay to the metastable ${ }^{2} E$ state with a lifetime of around $1.54 \mathrm{~ms}$ [25]. Using transitions from the ${ }^{2} E$ level to the ground state $\left({ }^{4} A_{2}\right)$, lasing at the sharp line around $680.4 \mathrm{~nm}$ could be obtained (three-level lasing mode). The same transition produces lasing at $694.3 \mathrm{~nm}$ in ruby [42].

The advantage of alexandrite in comparison with ruby is the lower energy difference between the ${ }^{2} E$ and ${ }^{4} T_{2}$ levels $\left(800 \mathrm{~cm}^{-1}\right.$ in alexandrite as opposed to approximately $2300 \mathrm{~cm}^{-1}$ in ruby), corresponding to only a few $\mathrm{kT}$ at room temperature $\left(200 \mathrm{~cm}^{-1}\right)$. Hence, a significant amount of the states in the ${ }^{4} T_{2}$ level is populated in the thermal quasiequilibrium after excitation $\left({ }^{2} E\right.$ acts as a storage level and increases its lifetime to around $262 \mu \mathrm{s}$ at room temperature). Moreover, duo to the Franck-Condon principle, transitions from the ${ }^{4} T_{2}$ level to ${ }^{4} A_{2}$ are favored over the transition from the ${ }^{2} E$ to ${ }^{4} A_{2}$. Hence, alexandrite can produce broadly tunable laser radiation in the $700-820 \mathrm{~nm}$ wavelength range as a fourlevel system $[\underline{26}, \underline{43}]$. This property also enables higher laser efficiencies at elevated temperatures above room temperature in alexandrite $[33,44]$. In contrast, the energy difference between the ${ }^{2} E$ and ${ }^{4} T_{2}$ states in ruby is too high to obtain reasonable population density in the ${ }^{4} T_{2}$ state.

One can directly pump alexandrite laser ions to the storage level (in-line pumping to level ${ }^{2} E$ ) to reduce the energy difference between the pump and lasing wavelengths and, hence, to minimize the quantum defect. The ${ }^{2} E$ level is actually an orbital doublet ( $R_{1}$ and $R_{2}$ lines), and the ${ }^{4} A_{2} \rightarrow R_{1 / 2}$ transitions (zero-phonon transitions) create two narrow (FWHM around $0.5 \mathrm{~nm}$ ) absorption peaks at 678.5 and $680.4 \mathrm{~nm}$ [24]. The inset graph in Fig. 1 shows the measured absorption spectrum of alexandrite in this region. Diverse excitation bands (broadband ${ }^{4} T_{1 / 2}$ excitations and narrowband $R_{1 / 2}$
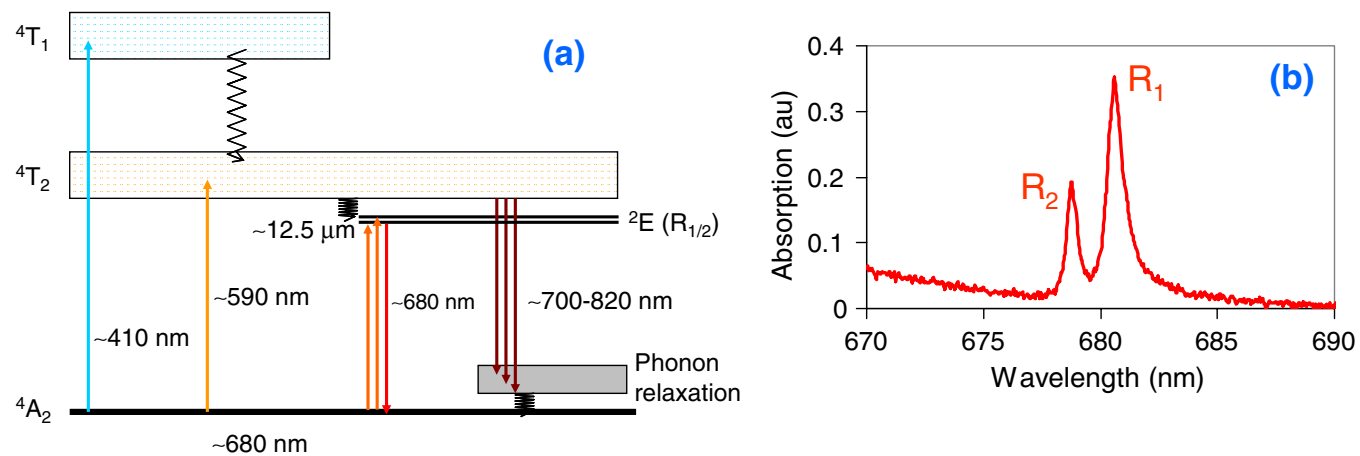

Fig. 1. (a) Simplified energy level diagram for the alexandrite gain medium. Lasing occurs between the vibronically broadened ${ }^{4} T_{2}$ and ${ }^{4} A_{2}$ levels. (b) Measured variation of the absorption coefficient as a function of wavelength for the transition from the ${ }^{4} A_{2}$ ground level to the doublet of the ${ }^{2} E$ level for the $E \| b$ axis (in absorption coefficient scale). 
excitations) allow different pump options for alexandrite. Flashlamps [45], Xe/Hg arc lamps [25,46], krypton ion lasers (647 nm) [47], dye lasers (615-680 nm) [36], frequencydoubled neodymium lasers around $530 \mathrm{~nm}[\overline{48,49]}$, and even sunlight [50] have been used for pumping $\mathrm{cw}$ alexandrite lasers. With lamp pumping, output powers as high as $60 \mathrm{~W}$ could be obtained. However, the systems are complex and bulky. Maximum reported slope efficiencies are 1.5\% (optical-tooptical with respect to incident pump power) $[25,46]$. On the other hand, single transverse-mode pump sources like dye lasers or argon ion lasers resulted in more efficient laser operation (up to 64\%) [36,47]. However, for these cases the pump source itself is an expensive laser system. Hence, none of these pump sources could provide the advantages of direct diode pumping and cannot compete with the Ti:sapphire lasers in the market.

Alexandrite lasers were first pumped by diodes in 1990, using two $5 \mathrm{~mW}$ single-mode diodes around $680.5 \mathrm{~nm}$ [51]. The alexandrite laser output power was less than $1 \mathrm{~mW}$ [51]. The same group has then pumped alexandrite lasers using two $250 \mathrm{~mW}$ multimode diodes around $640 \mathrm{~nm}$ and obtained $25 \mathrm{~mW}$ of cw output power [36]. In a study published in 2005, using a high-power $(10 \mathrm{~W})$ diode array consisting of many single-emitter multimode diodes around $680 \mathrm{~nm}$, cw output powers as high as $1.3 \mathrm{~W}$ have been obtained from alexandrite lasers [52]. However, the transverse intensity profile of the emission from the diode array was quite structured. This resulted in a multimode laser output ( $\mathrm{M}^{2}$ of 2.8), as well as relatively low slope efficiencies. Recently, Damzen et al. obtained cw output powers as high as $6.4 \mathrm{~W}$ from an alexandrite laser system side-pumped by two $29 \mathrm{~W}$ diode arrays at $635 \mathrm{~nm}$ [53]. In this study, the slope efficiencies remained below $20 \%$.

In this work, to our knowledge, we present the first demonstration of high-brightness TDLs as pump sources for the alexandrite gain medium. The TDL provided up to $1 \mathrm{~W}$ of output power with a tunable optical spectrum centered around 678.5 or $680.4 \mathrm{~nm}$, which is suitable for in-line pumping of alexandrite by populating the R-lines. The diodes possess $\mathrm{M}^{2}\left(1 / e^{2}\right)$ values of 1.1 in the fast axis and 5 in the slow axis, and a brightness of about $500 \mathrm{~mW} / \mu \mathrm{m}^{2}$, which represents a twofold improvement compared to the state-of-the-art singlemode and broad-stripe single-emitter diodes in the same wavelength range [54]. While pumping at the $678.5 \mathrm{~nm}$ line, using a $0.5 \%$ output coupler, we have obtained output powers as high as $168 \mathrm{~mW}$, slope efficiency as high as 38\%, and a cw tuning range from 724 to $816 \mathrm{~nm}$. The laser output was multimode with $\mathrm{M}^{2}$ values of 1.25 and 4.5 in the horizontal and vertical axes, respectively. Upon insertion of a slit near the output coupler, we could also obtain a beam profile close to $\mathrm{TEM}_{00}$ at an output power of $112 \mathrm{~mW}$. Using Findlay-Clay analysis, the round-trip loss of the cavity was estimated to be around $0.4 \%$. Caird analysis gave a value of $0.3 \%$ for the round-trip loss and $63 \%$ for the intrinsic slope efficiency. Last, upon pumping at the $680.4 \mathrm{~nm}$ line with a stronger absorption, cw output powers as high as $200 \mathrm{~mW}$ could be obtained. These results demonstrate the suitability of recently available tapered diode (TD) technology as efficient pump sources for alexandrite lasers. This initial study has the potential to pave the way for the development of low-cost compact femtosecond alexandrite lasers near $750 \mathrm{~nm}$ as optimal pump sources for multiphoton microscopy.

The paper is organized as follows. Section 2 introduces the experimental setup and methods employe $\bar{d}$. Section $\underline{3}$ presents detailed cw lasing results. In Section $\underline{4}$, we summarize the results and provide a general discussion.

\section{EXPERIMENTAL SETUP}

We start this section with a brief description of the TD that was employed as a pump source for alexandrite. We have been using similar TDs to pump Cr:LiCAF and Cr:LiSAF gain media to minimize the complexity in pumping geometry [54]. Similarly, we believe that the TDs that provide the highest brightness output at these wavelengths will considerably simplify the complexity and lower the cost of cw alexandrite lasers. The TDs used in this study were grown and characterized at the facilities of Ferdinand Braun Institute [55]. The TD has a total cavity length of $2 \mathrm{~mm}$. It consists of a $500 \mu \mathrm{m}$ long ridge waveguide section and a $1.5 \mathrm{~mm}$ long tapered amplifier section with a flared angle of $3^{\circ}$. At the output the TD has an aperture with a width of $80 \mu \mathrm{m}$. The front facet and rear facets of the TD have reflectivities of $1 \%$ and $94 \%$, respectively. The TD is mounted on standard C-mounts, with p-side down with copper tungsten submounts using AuSn solder. A commercial c-mount diode fixture (ILX LDM-4409) with thermoelectric cooling is used for housing the diode.

Figure 2(a) shows the measured variation of the optical output power of the TD, with drive current at diode holder temperatures of $15^{\circ} \mathrm{C}, 20^{\circ} \mathrm{C}$, and $25^{\circ} \mathrm{C}$. At $15^{\circ} \mathrm{C}$, the TD has a lasing threshold of $500 \mathrm{~mA}$ and a slope efficiency of $0.75 \mathrm{~W} / \mathrm{A}$. At a drive current of $2 \mathrm{~A}$, the diode provides up to $1100 \mathrm{~mW}$ of
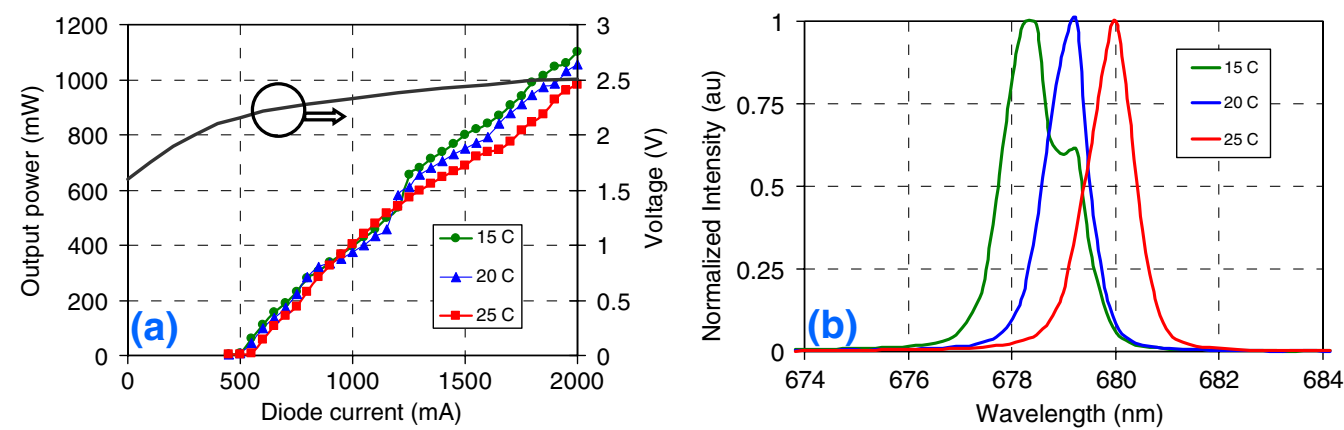

Fig. 2. (a) Measured variation of optical output power with input current for the TDL at diode holder temperatures of $15^{\circ} \mathrm{C}, 20^{\circ} \mathrm{C}$, and $25^{\circ} \mathrm{C}$. Corresponding voltage values of the TD for $25^{\circ} \mathrm{C}$ operation are also shown. (b) Measured optical spectra of the TD at $2 \mathrm{~A}$ diode current and at the diode holder temperatures of $15^{\circ} \mathrm{C}, 20^{\circ} \mathrm{C}$, and $25^{\circ} \mathrm{C}$. By adjusting diode current and temperature, it is possible to tune the central wavelength of emission to the absorption peaks at 678.5 and $680.4 \mathrm{~nm}$. 

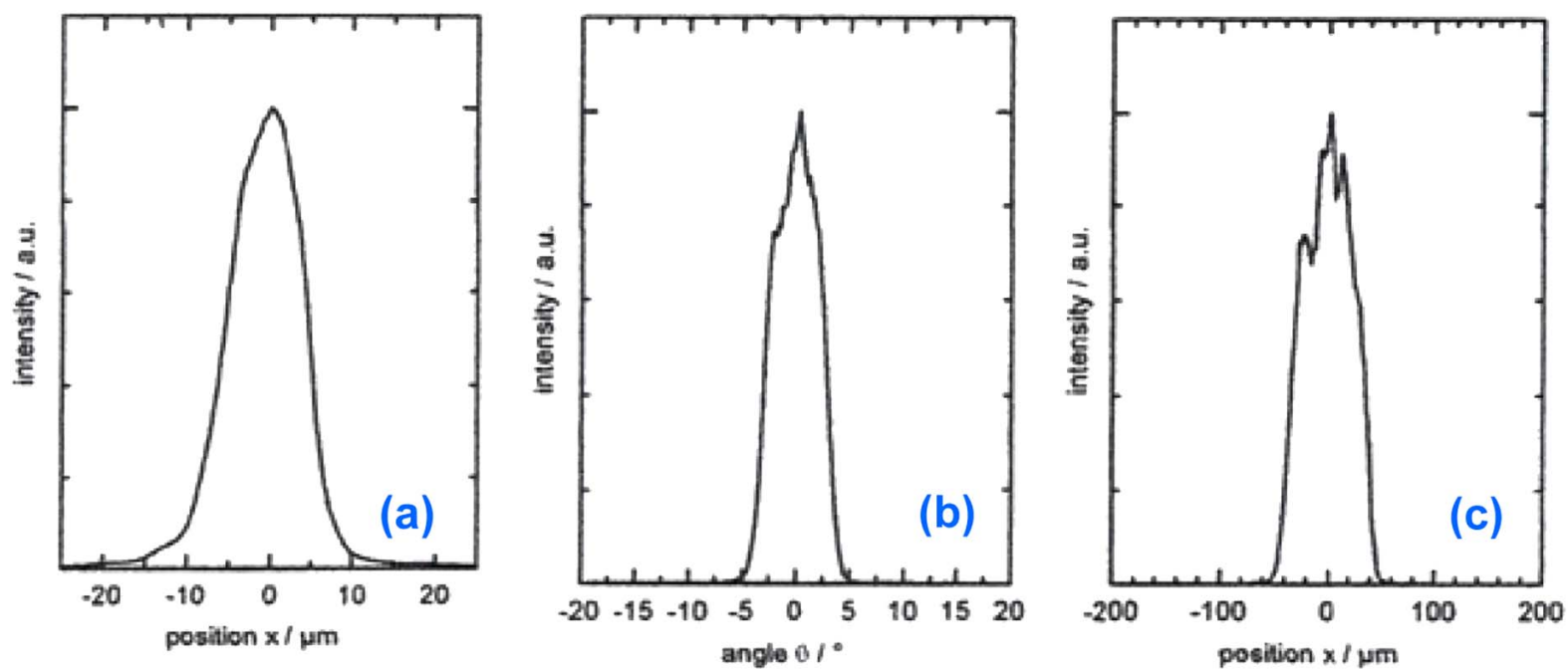

Fig. 3. (a) Beam waist, (b) far-field, and (c) near-field profiles for the slow axis of the TDL measured at $1 \mathrm{~W}$ of output power and at a cooling temperature of $15^{\circ} \mathrm{C}$.

output power, with a diode voltage of $2.5 \mathrm{~V}$, which corresponds to an electrical-to-optical conversion efficiency of about $22 \%$. The diode spectrum is centered at $678.5 \mathrm{~nm}$ and has a width of about $1.5 \mathrm{~nm}(\mathrm{FWHM})$ at $15^{\circ} \mathrm{C}$ case temperature and 2 A diode current. We note here that this wavelength directly matches the $R_{2}$ line of the alexandrite crystal. As we can see from Fig. 2(b), by increasing the diode case temperature to $25^{\circ} \mathrm{C}-30^{\circ} \mathrm{C}$, it was possible to shift the diode wavelength to the $R_{1}$ line of alexandrite, which actually has a stronger absorption [24] [Fig. 1(b)]. However, the diode output power is lower for this case $\left(980 \mathrm{~mW}\right.$ at $2 \mathrm{~A}$ and $\left.25^{\circ} \mathrm{C}\right)$ due to the increased temperature. In most of our lasing experiments we have exploited the $R_{2}$ line for pumping. For comparison, lasing results obtained while pumping the $R_{1}$ transition will be presented in Fig. 11.

The method of the moving slit (ISO Standard 11146, Annex A) is applied to measure the beam quality of the TD. The intensity profiles of the beam waist, the intensity distribution along the front facet, and the astigmatism of the diode output are also investigated. During the measurements, the laser spot is magnified by using a telescope with a magnification of about 60 . The studies are carried out at a diode output power of $1 \mathrm{~W}$ and at diode holder temperature of $15^{\circ} \mathrm{C}$. The beam waist, far-field, and near-field profiles of the TDL in the slow axis are shown in Fig. 3. Around $96 \%$ of the emitted power originates from the central lobe of the beam waist. We have found a beam waist of $16.4 \mu \mathrm{m}$ at the $1 / e^{2}$ level, and a farfield angle of $7.5^{\circ}\left(1 / e^{2}\right)$, which leads to a beam propagation ratio of $5\left(1 / e^{2}\right)$. In the fast axis, the beam propagation ratio is determined to be better than 1.1 at the $1 / e^{2}$ level, and the beam divergence was about $30^{\circ}$. The astigmatism of the diode is measured to be $650 \mu \mathrm{m}$. Using the above-mentioned parameters, the brightness of the laser device is estimated as $B=500 \mathrm{~mW} / \mu \mathrm{m}^{2}$, which is about 2 times higher than the commercial single-mode diodes and single-emitter devices on the market. However, it is possible to grow TDs with brightness approaching $1000 \mathrm{~mW} / \mu^{2}$ [54,55]. Hence, the results presented in this study are likely to be improved in the near future by using even higher quality TDs.

Figure 4 shows a schematic of the TD-pumped alexandrite laser. The diode output beam is first collimated with a $4.5 \mathrm{~mm}$ focal length aspheric lens with a numerical aperture of 0.54 (f1 in Fig. 4). A cylindrical lens with a focal length of $50 \mathrm{~mm}$ (fz, acting on the fast axis) matched the diode divergences in both axes to minimize astigmatism. Then the pump beam is focused inside the alexandrite crystal, using an achromatic doublet with a focal length of $60 \mathrm{~mm}$ (f2). A half-wave plate (HWP) and a polarizing beam splitter cube (PBS) are used to adjust the incident pump power on the crystal. Variation of the diode current changes the output wavelength. Therefore, the diode current is kept at $2 \mathrm{~A}$ in all the experiments. The incident pump power on the crystal at the maximum drive current has been $925 \mathrm{~mW}$ (lower than $1.1 \mathrm{~W}$ due to the transmission losses of the optics). The $\mathrm{cw}$ laser experiments are based on an astigmatically compensated X-cavity consisting of two curved mirrors (M1 and M2, ROC $=75 \mathrm{~mm}$ ), a flat end high reflective (HR) mirror, and a flat output coupler (OC). The HR mirrors have a reflectivity greater than $99.9 \%$ from 725 to $925 \mathrm{~nm}$ and a pumping window with transmission above $98 \%$ in the red spectral region. The length of the long cavity arm has been adjusted to $45 \mathrm{~cm}$ to obtain a beam waist of approximately $20 \mu \mathrm{m}$ inside the gain medium. The short arm length is $35 \mathrm{~cm}$ long in all the experiments. A Brewster-cut, 10-mm-long, alexandrite crystal (Northrop Grumman) with a thickness of $3 \mathrm{~mm}$ and a width of $5 \mathrm{~mm}$ (chromium-doping level $0.2 \%$ ) is used in the study. The crystal absorbs $73 \%$ and $95 \%$ of the incident TM polarized pump light at 678.5 and $680.4 \mathrm{~nm}$, respectively. The crystal is mounted with indium foil in a copper holder, which is temperature stabilized to $25^{\circ} \mathrm{C}$

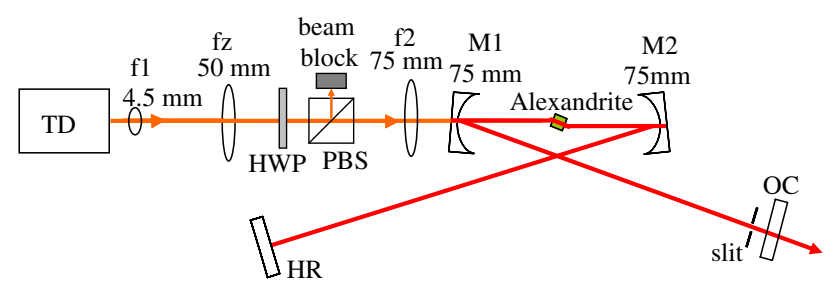

Fig. 4. Schematic of the cw alexandrite oscillator pumped by a TDL (TD). M1, M2, curved pump mirrors with a ROC of $75 \mathrm{~mm}$; OC, output coupler; f1, collecting and collimating aspheric lens; fz, cylindrical collimating lens (matches the divergence in both axes); $\mathrm{f} 2$, focusing lens; HWP, half-wave plate; PBS, polarizing beam splitter cube. 


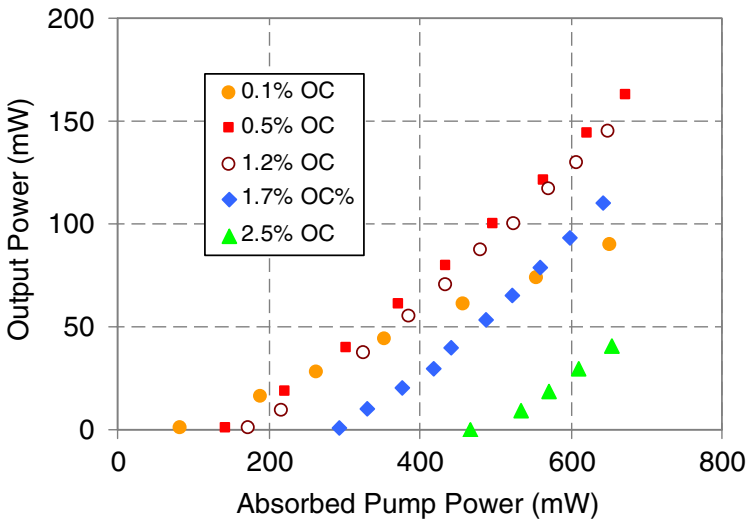

Fig. 5. Measured cw output power of the TD pumped alexandrite laser as a function of the absorbed pump power at various levels of output coupling between $0.1 \%$ and $2.5 \%$. The laser was pumped at the $678.4 \mathrm{~nm}\left(R_{2}\right)$ line and the crystal absorbed $73 \%$ of the incident pump power. The data were taken without an intracavity slit and the laser output was slightly multimode.

with a circulating water chiller. We note that we have observed a strong tendency of alexandrite lasers for self-pulsing (also known as self-Q-switching) [56]. Similar phenomena have also been reported in other $\mathrm{Cr}^{+3}$-doped materials, such as Cr:LiCAF [56], Cr:LiSAF [57,58], and ruby [59-62]. However, it is possible to obtain pure cw operation by fine adjustment of the separation of the curved mirrors.

\section{RESULTS AND DISCUSSION}

Figure 5 shows the measured efficiency curves of the cw alexandrite laser using several different output couplers with transmission ranging from $0.1 \%$ to $2.5 \%$. The highest power was obtained using a $0.5 \%$ output coupler. With this OC, the laser produced $168 \mathrm{~mW}$ at an absorbed pump power level of $670 \mathrm{~mW}$. The free-running laser wavelength was $755 \mathrm{~nm}$. The laser slope efficiency was $25 \%$ at low incident power levels and then increased to $38 \%$ at higher pump powers (an overall fit gave an average slope efficiency of $31 \%$ ). This unusual effect is clearly visible in Fig. 5 . We believe that this is possibly due to an improvement in mode matching between the cavity and pump modes at increased pump power levels. The overall optical-to-optical and electrical-to-optical conversion efficiencies were $\sim 15 \%(168 \mathrm{~mW} / 1100 \mathrm{~mW})$ and $\sim 3.4 \%(168 \mathrm{~mW} / 5 \mathrm{~W})$, respectively. We note here that, while using the $0.5 \%$ output coupler, $30 \mathrm{~mW}$ of total laser power was found to be leaking from the cavity high reflectors (a total of five bounces, two bounces from M1, two bounces from M2, and one bounce from HR, Fig. 4). With the $0.5 \%$ output coupler, the estimated intracavity power is $32.6 \mathrm{~W}$. This finding shows that the cavity mirrors have a leakage loss of about $0.1 \%(30 \mathrm{~mW} / 32.6 \mathrm{~W})$, corresponding to a leakage loss level of $0.02 \%$ per bounce. Using high reflectors with improved coating, the obtained output power can exceed $200 \mathrm{~mW}$ from this configuration.

Figure 6 demonstrates the measured variation of lasing threshold as a function of the output coupler transmission. Variation of maximum obtainable power with output coupler transmission is also shown in the same graph (at the maximum absorbed pump power of $670 \mathrm{~mW}$ ). First of all, the optimum output coupling is $0.5 \%-1 \%$, which highlights the low-gain nature of the material. This fact also underlines

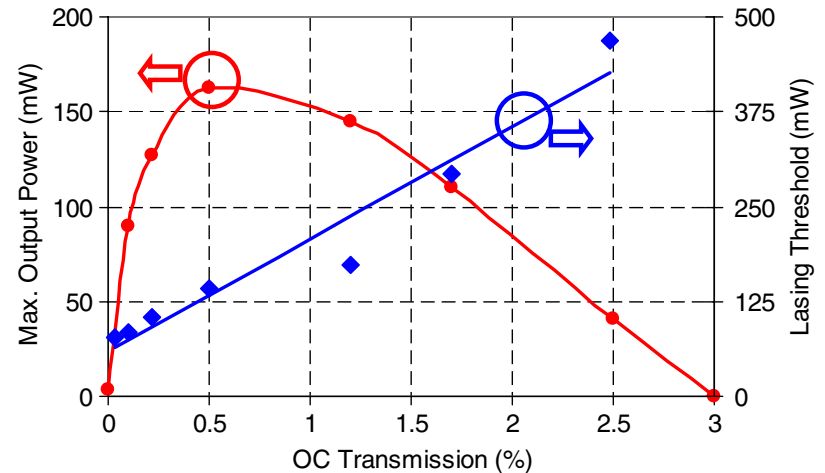

Fig. 6. Measured variation of lasing threshold and maximum obtainable output power with the output coupler transmission. Using Findlay-Clay analysis, the round-trip passive cavity loss (L) was estimated to be $(0.4 \pm 0.1) \%$.

the importance of using high-quality crystals and mirrors with low passive losses in the resonator to attain high slope efficiencies.

The measured lasing threshold was $143 \mathrm{~mW}$ with the $0.5 \%$ output coupler (optimum output coupling, Fig. 6). A lasing threshold of $83 \mathrm{~mW}$ was measured using a $0.1 \%$ output coupler. Moreover, operating a cavity with all HR mirrors achieved a lasing threshold of $78 \mathrm{~mW}$. Using the measured variation of lasing threshold $\left(P_{\text {th }}\right)$ with output coupling, it is possible to estimate the round-trip laser resonator losses (L) at the lasing wavelength $(\sim 755 \mathrm{~nm})$. According to Findlay-Clay analysis $[\underline{17}, \underline{63}], P_{\text {th }}$ is linearly proportional to $L$, according to

$$
P_{\mathrm{th}}=\frac{\pi\left(w_{p}^{2}+w_{c}^{2}\right) h \nu_{p}}{4\left(\sigma_{e}-\sigma_{\mathrm{ESA}}\right) \tau_{f} \eta_{p}}\left(2 A_{g}+T+L\right)
$$

In Eq. (1), $h$ is Planck's constant, $v_{p}$ is the pump photon frequency, $\eta_{p}$ is pumping efficiency, $w_{p}\left(w_{c}\right)$ is the pump (cavity) beam waists, $\sigma_{e}\left(\sigma_{\mathrm{ESA}}\right)$ is the emission (excited state absorption) cross section, $\tau_{f}$ is the fluorescence lifetime of the upper laser level, $A_{g}$ is the ground state absorption of the $\mathrm{Cr}^{3+}$ ions, and $T$ is the transmission of the output coupler. Selfabsorption losses due to the $\mathrm{Cr}^{3+}$ ion are insignificant around $755 \mathrm{~nm}$. Consequently, $A_{g}$ can be ignored in Eq. 1, which allows simplification of Eq. 1 to $P_{\text {th }}=C(T+L)$, where $C$ is a constant that includes all the initial terms. We have determined the best linear fit to the experimental data as $P_{\text {th }} \approx 60+$ $148 T$ (in milliwatt units, solid line in Fig. 6). From this fit, the total round-trip cavity loss $(\mathrm{L})$ is estimated to be $(0.4 \pm 0.1) \%$.

The measured lasing threshold values with alexandrite are higher than what we have achieved earlier with TD pumped Cr:colquiriite lasers. For example, using a $0.5 \%$ output coupler, we have measured lasing thresholds of 45 and $93 \mathrm{~mW}$ from Cr:LiSAF and Cr:LiCAF lasers, respectively (143 mW was measured with alexandrite). We believe that this is partly because alexandrite possesses a smaller emission crosssection-lifetime product $\left(\sigma_{\mathrm{em}} \tau, \sigma_{\mathrm{em}}=\right.$ stimulated emission cross section, and $\tau=$ room-temperature fluorescence lifetime). As can be seen from Eq. (1), $P_{\text {th }}$ is inversely proportional to $\sigma_{\mathrm{em}} \tau$. Due to the presence of the storage effect of the ${ }^{2} E$ energy level, alexandrite has a relatively long roomtemperature fluorescence lifetime (262 $\mu \mathrm{s}$ [25]). However, its emission cross section is quite low $\left(0.7 \times 10^{-20} \mathrm{~cm}^{2}\right)$ at 
room temperature, resulting in a $\sigma_{\mathrm{em}} \tau$ product of $183 \mu \mathrm{s} \times$ $10^{-20} \mathrm{~cm}^{2}$ [25]. This value is higher than Ti:sapphire $(131 \mu \mathrm{s} \times$ $\left.10^{-20} \mathrm{~cm}^{2}[\overline{34}]\right)$, which is also known for its high lasing thresholds, but lower than Cr:LiCAF $\left(228 \mu \mathrm{s} \times 10^{-20} \mathrm{~cm}^{2}\right.$ [34]) and Cr:LiSAF $\left(322 \mu \mathrm{s} \times 10^{-20} \mathrm{~cm}^{2}\right.$ [34]). We also note here that the beam quality of the TD in this study $\left(\mathrm{M}_{\text {slow }}^{2} \sim 5\right)$ was worse than what has been used earlier in pumping Cr:LiCAF/LiSAF lasers $\left(\mathrm{M}_{\text {slow }}^{2} \sim 2.5[\underline{54}]\right)$.

Due to the multimode nature of the pump beam in the slow axis, the alexandrite laser output was also multimode in the corresponding axis. Figure 7 shows the measured position dependence of the spot size function of the alexandrite laser near the focus of a $6 \mathrm{~cm}$ lens. The best fit value for $\mathrm{M}^{2}$ was determined to be 1.25 and 5 in the $\mathrm{x}$ and $\mathrm{y}$ axes, respectively. We note here that, despite the multimode pump beam, it was possible to push the alexandrite to operate with a $\mathrm{TEM}_{00}$ mode. To this end, we have inserted a circular slit near the output coupler and decreased the aperture size until the laser produced a circular beam profile. The inserted slit generates a high level of losses for the higher-order modes and the laser power predominantly appeared in the fundamental mode (Fig. 8). However, this has a negative effect on the mode matching between the pump and cavity modes. Hence, with the insertion of the slit, using the $0.5 \%$ output coupler, the laser slope efficiency decreased from $38 \%$ to $25 \%$ and the obtained output powers decreased from 168 to $112 \mathrm{~mW}$.

We have also used the measured variation of the slope efficiency with output coupler transmission (Fig. 9) to estimate the resonator losses at the lasing wavelength (Caird analysis [64]). According to this method, the slope efficiency $(\eta)$ of a laser can be expressed as

$$
\eta=\left[\left(\frac{h v_{l}}{h v_{p}}\right) \eta_{p}\left(\frac{\sigma_{e}-\sigma_{\mathrm{ESA}}}{\sigma_{e}}\right)\right] \frac{T}{T+L}=\eta_{0} \frac{T}{T+L},
$$

where $v_{l}$ is the laser photon frequency and $\eta_{0}$ is the maximum (intrinsic) slope efficiency that can be obtained at high output coupling. Least-squares fitting to the experimentally measured data gave best-fit $L$ and $\eta_{0}$ values of $(0.3 \pm 0.1) \%$ and $(63 \pm 5) \%$, respectively. We note here that in Caird analysis, we have considered only the slope efficiency data taken with $0.1 \%, 0.2 \%$, and $0.5 \%$ output couplers (filled squares in Fig. 9 ) and ignored the data taken with $1.2 \%, 1.7 \%$, and $2.5 \%$ output

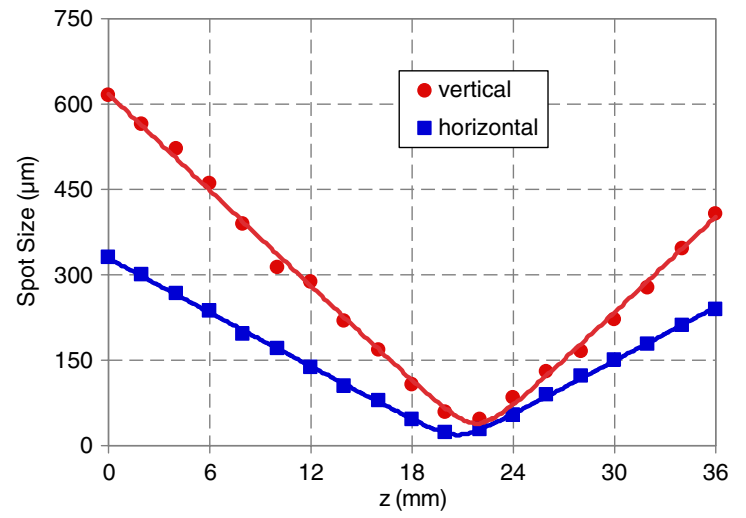

Fig. 7. Measured position dependence of the spot size $\left(1 / e^{2}\right)$ function from the alexandrite laser near the focus of a $6 \mathrm{~cm}$ lens in the $z$ and $y$ axes. Least-squares fitting to the experimental data gave an $\mathrm{M}^{2}$ value of 1.25 and 4.5 for the horizontal and vertical axes, respectively.

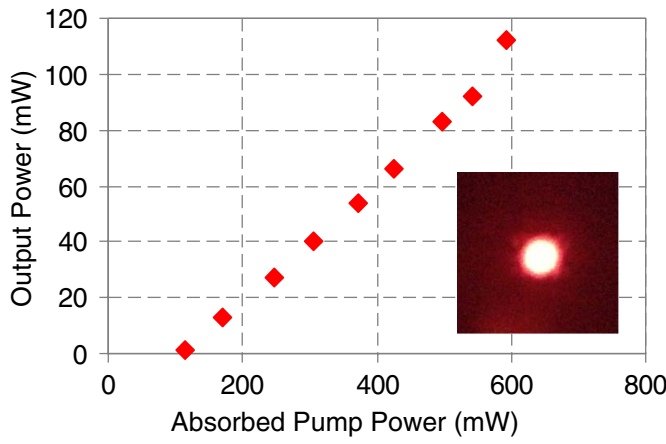

Fig. 8. Continuous-wave output power versus absorbed pump power for the TD pumped alexandrite laser taken using a $0.5 \%$ output coupler and the intracavity slit. The laser is pumped at the $678.5 \mathrm{~nm}\left(R_{2}\right)$ line and the crystal absorbed $73 \%$ of the incident pump power. The laser output was single mode (inset figure shows the measured beam profile).

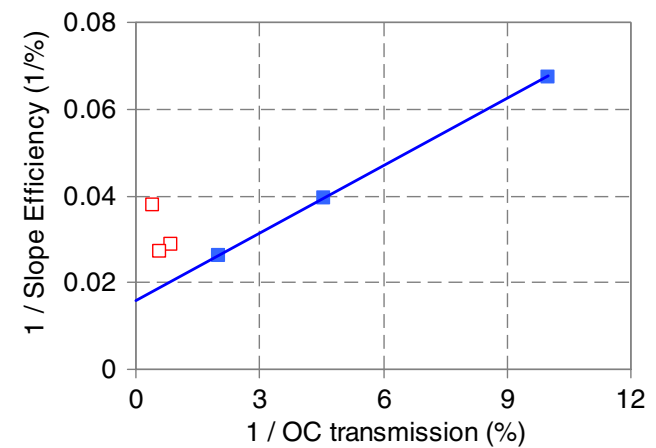

Fig. 9. Measured variation of the inverse of the slope efficiency as a function of the inverse of the output coupling. Using Caird analysis, the round-trip passive cavity loss $(\mathrm{L})$ and the intrinsic slope efficiency $\left(\eta_{0}\right)$ were determined to be $(0.3 \pm 0.1) \%$ and $(63 \pm 5) \%$, respectively. Note that some of the experimental points were excluded in the analysis (empty rectangles).

couplers (empty squares in Fig. 9). In conflict with theory, the obtained slope efficiencies at high output coupling would start to decrease. A similar effect is also observed in Cr:colquiriites, where it is due to the presence of Auger energy upconversion processes [2]. Here, the decrease in slope efficiency is a side

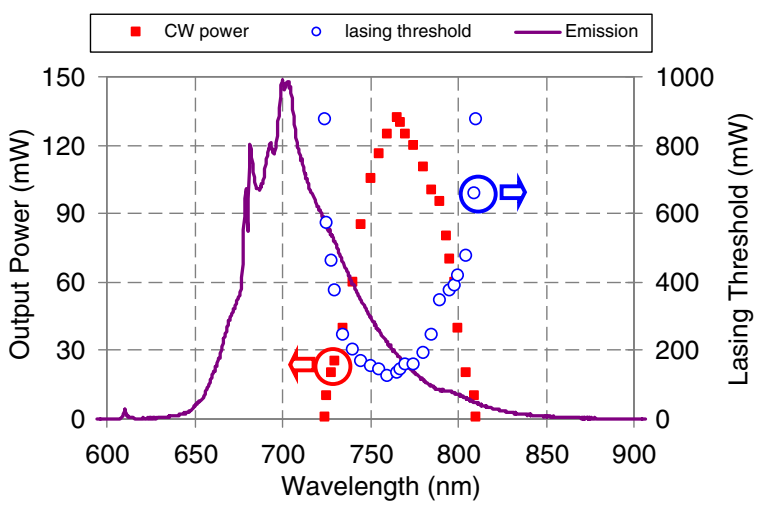

Fig. 10. Continuous-wave tuning curve of the alexandrite laser taken with the $0.5 \%$ output coupler, at an absorbed pump power of $670 \mathrm{~mW}$. The laser could be tuned smoothly from 725 to $810 \mathrm{~nm}$. The measured variation of lasing threshold with wavelength and room-temperature emission spectrum $(E \| b)$ of the alexandrite gain medium are also shown. 


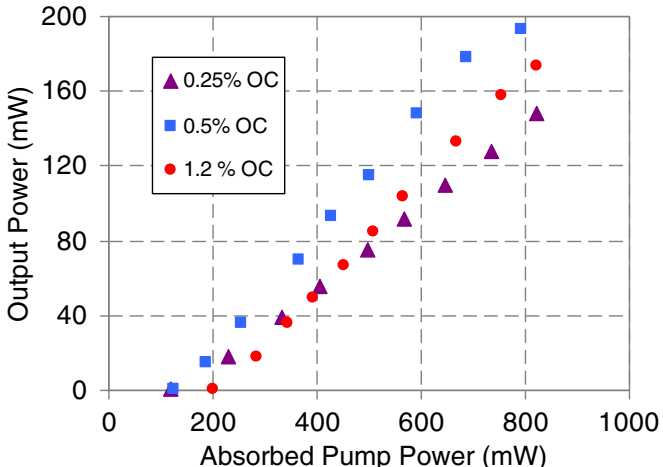

Fig. 11. Measured cw output power as a function of the absorbed pump power for the TD-pumped alexandrite laser taken at $0.25 \%$, $0.5 \%$, and $1.2 \%$ output coupling. The laser is pumped at the $680.5 \mathrm{~nm}$ $\left(R_{1}\right)$ line and the crystal absorbed $95 \%$ of the incident pump power. The data were taken without an intracavity slit and the laser output was slightly multimode.

effect of increasing lasing threshold due to the decrease in fluorescence lifetime. We suspect that a similar mechanism might also be at work in the alexandrite gain medium. More details on the effect of Auger upconversion on laser slope efficiency can be found in [2].

The cavity loss estimate using Findlay-Clay (0.4 $\pm 0.1 \%)$ and Caird $(0.3 \pm 0.1 \%)$ analyses are in relatively good agreement with each other. As we have discussed earlier, due to the nonoptimum reflectivity bandwidths of the HR mirror set that was used in this study, we had about $0.1 \%$ leakage loss from the HR mirrors. Other parasitic losses of the HR mirrors are specified to be about $0.005 \%$, so we estimate that an additional loss of $0.05 \%$ will be present from all the bounces on the cavity optics (four bounces on the curved high reflector mirrors, one bounce on each of the flat cavity high reflector and the output coupler). The remaining $0.2 \%$ loss should be attributed to the alexandrite crystal, where in each pass one roughly gets a loss of $0.1 \%$. For the 10 -mm-long alexandrite crystal, this corresponds to a quite low loss level of only about $0.1 \%$ per centimeter $(\mathrm{cm})$, which is similar to what can be achieved with Cr:LiCAF (0.14\% per cm [2]) and Cr:LiSAF (0.2\% per $\mathrm{cm}$ [65]), and much better than typical Ti:sapphire crystals (2\% per $\mathrm{cm}[11])$. This also shows itself in the crystal figure of merit (FOM) value, which is defined as the ratio of the absorption coefficient at the pump wavelength to the absorption coefficient at the lasing wavelength. The alexandrite crystal that was used in this study has an estimated FOM of about

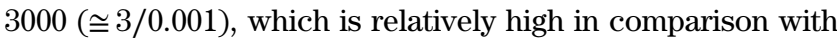
Cr:LiCAF (2150 [2]), Cr:LiSAF (1500 [65]), and Ti:sapphire (150 [11]]). On the other hand, Scheps et al. reported a passive loss level of $0.06 \%$ per $\mathrm{cm}$ from a $0.2 \%$ chromium-doped alexandrite crystal (the crystal probably had even lower losses since they have assumed that the cavity optics did not contribute to the resonator losses) [36]. Hence, the slope efficiencies that were reported in this study could be further improved by using better quality alexandrite crystals and cavity optics.

Figure 10 shows the measured cw tuning range of the alexandrite laser using the $0.5 \%$ output coupler. For comparison, we have also included the emission spectrum of the material (note the sharp $R$ lines that were used for pumping). Figure 10 also includes the measured variation of lasing threshold with wavelength. The laser wavelength was tuned by (i) inserting an SF11 prism into the HR arm or by (ii) inserting a birefringent tuning plate. Both methods give similar results in terms of tuning range and output power, but the spectra were narrower with the birefringent plate $(0.5 \mathrm{~nm}$ FWHM). With the $0.5 \%$ output coupler, the alexandrite laser could be tuned smoothly from 724 to $810 \mathrm{~nm}(86 \mathrm{~nm})$. When we replaced the output coupler with a high reflector, the long wavelength edge could be extended up to $816 \mathrm{~nm}$. We believe tuning below $724 \mathrm{~nm}$ was prevented due to the increased passive losses of the cavity high reflectors, which had reflectivity above $99.9 \%$ from 725 to $925 \mathrm{~nm}$. On the long wavelength side, the mirrors had good reflectivity, but the emission cross section of the material becomes too low to have sufficient gain above $816 \mathrm{~nm}$

Table 2. Summary of Results from Literature for cw Alexandrite Lasers ${ }^{a}$

\begin{tabular}{|c|c|c|c|c|c|c|c|}
\hline Pump Source & $\begin{array}{c}\text { Pump Wavelength } \\
\text { (nm) }\end{array}$ & $\begin{array}{l}\text { Pump Power } \\
\text { (W) }\end{array}$ & $\begin{array}{c}\text { Laser Power } \\
(\mathrm{mW})\end{array}$ & $\begin{array}{c}\text { Slope Efficiency } \\
(\%)\end{array}$ & $\begin{array}{l}\text { Laser Output } \\
\left(\mathrm{M}^{2} \text { value }\right)\end{array}$ & Year & Ref. \\
\hline Flashlamp & Broadband & 3000 & 6500 & 0.8 & Multimode & 80 & {$[\underline{45}]$} \\
\hline Xe arc lamp & Broadband & 8000 & 20,000 & 0.5 & Multimode & 85 & [25] \\
\hline Xe arc lamp & Broadband & - & 2000 & - & TEM00 & 85 & {$[25]$} \\
\hline Krypton laser & 647 & 1.6 & 600 & 51 & - & 83 & {$[\underline{47}]$} \\
\hline Sun-light & Broadband & $\sim 1000$ & 12,000 & 1.2 & - & 99 & {$[50]$} \\
\hline SH of Nd:YAG & 532 & 0.215 & 11 & 10.5 & - & 05 & {$[\underline{48}]$} \\
\hline SH of Nd:YVO4 & 532 & 5 & 1420 & 36 & TEM00 & 06 & {$[\overline{49}]$} \\
\hline Single-mode diode & 680.4 & $2 \times 0.005$ & - & 25 & - & 90 & {$[\overline{51}]$} \\
\hline Multimode diode & 640 & $2 \times 0.25$ & 25 & 28 & - & 93 & {$[\underline{36}]$} \\
\hline Multimode diode array & 680.4 & 10 & 1300 & 24 & 2.8 & 05 & {$[\overline{52}]$} \\
\hline
\end{tabular}

${ }^{a}$ For comparison, pump source, pump wavelength, pump power, laser power, laser slope efficiency, and laser beam quality have been specified for each case. Results obtained under diode pumping have been listed at the bottom of the table.

${ }^{b}$ Denotes the experimental results obtained in this work. 
In earlier studies, using flashlamp pumping, Walling et al. demonstrated tuning of a pulsed alexandrite laser from 701 to $818 \mathrm{~nm}$ [24]. Pulsed pumping with high lamp energies $(100 \mathrm{~J})$ and use of a lower Cr doping of the crystal $(0.043 \%)$ was effective in reaching the short wavelength $(701 \mathrm{~nm})$ limit in that study [24]. Later, a tuning range extending up to $858 \mathrm{~nm}$ was reported by Kuper et al. from a flash lamp pumped-pulsed alexandrite laser at elevated temperatures above room temperature [33]. With temperature, the emission profile of the alexandrite shifts to longer wavelengths and the emission cross section also increases due to the increased role of the storage level [24,33]. In particular, reaching the $858 \mathrm{~nm}$ range required an alexandrite rod temperature of $513^{\circ} \mathrm{C}$ and a pump energy of $105 \mathrm{~J}$ [33].

So far, we have presented cw laser results of the alexandrite laser taken while pumping at the $678.5 \mathrm{~nm}$ line. For this case, the incident pump power was $925 \mathrm{~mW}$, the absorption of the alexandrite crystal was $73 \%$, and the total absorbed pump power was $675 \mathrm{~mW}$. As mentioned earlier, by adjusting the diode temperature, it was possible to tune the TD wavelength to the other $R$ line $(680.5 \mathrm{~nm})$. At this line, the incident pump power on the crystal decreased to $865 \mathrm{~mW}$. However, the crystal absorption climbed to $95 \%$, resulting in an overall absorbed pump power of $820 \mathrm{~mW}$. Therefore, we expect a rise in the obtainable output powers from the alexandrite crystal. The efficiency curves taken with $0.25 \%, 0.5 \%$, and $1.2 \%$ output couplers are shown in Fig. 11. Using a $0.5 \%$ output coupler, we measured output powers as high as $195 \mathrm{~mW}$. The lasing threshold values and the slope efficiencies remained almost the same as those obtained in the case of $678.5 \mathrm{~nm}$ pumping.

\section{CONCLUSIONS AND SUMMARY}

To provide a comparison with earlier work, Table $\underline{2}$ represents a summary of the published laser results for $\mathrm{cw}$ alexandrite lasers in the literature. Results obtained in this work have also been listed at the end of the table (marked with "b"). As mentioned earlier, the broad emission bands of alexandrite in the visible enables use of a variety of pump sources, including flash lamps [45], Hg and Xe lamps [25, $\underline{46}, \underline{66}]$, sunlight [50], and the second harmonic of Nd-based systems [48,49]. Using these pump sources, tens of Watts of cw output power have been obtained from alexandrite lasers. However, these pump sources are complicated, which limits the usability of alexandrite lasers. On the other hand, there are only a few studies that report diode-pumped cw alexandrite lasers.

Earlier studies on diode pumping of $\mathrm{cw}$ alexandrite lasers have used low-brightness sources that were available at the time, resulting in limited levels of output power [36, $\underline{51}$ ]. Later studies using high-power diode arrays as pump sources resulted in the generation of cw output powers as high as $1.3 \mathrm{~W}$ [52] and $6.4 \mathrm{~W}$ [53]. Here, we have reported pumping of cw alexandrite lasers with high-brightness TDLs for the first time, to our knowledge. Moreover, this is probably the first report of pumping alexandrite via the $R_{2}$ transition at $678.5 \mathrm{~nm}$. The obtained slope efficiencies are the highest achieved so far from diode-pumped cw alexandrite lasers and the obtained output powers (200 $\mathrm{mW}$ ) are also sufficient for many applications. We also note here that there is great potential for future improvements by using better quality alexandrite crystals and cavity optics, as well as higher brightness TDs. Our present results clearly show that the recent advances in TD technology are key factors that result in the development of low-cost, compact, and efficient $\mathrm{cw}$ alexandrite lasers with moderate output power levels.

\section{ACKNOWLEDGMENTS}

We thank Michael Schmalz, Christian Beschle, and Adnan Kurt for help in the experiments. We acknowledge support from the Scientific and Technological Research Council of Turkey (TÜBİTAK, 113F199), a European Union Marie Curie Career Integration grant (PCIG11-GA-2012-321787), and the Alexander von Humboldt Foundation.

\section{REFERENCES}

1. A. A. Lagatsky, C. T. A. Brown, and W. Sibbett, "Highly efficient and low threshold diode-pumped Kerr-lens mode-locked Yb:KYW laser," Opt. Express 12, 3928-3933 (2004).

2. U. Demirbas, R. Uecker, D. Klimm, and J. Wang, "Low-cost, broadly tunable (375-433 nm \& 746-887 nm) Cr:LiCAF laser pumped by one single-spatial-mode diode," Appl. Opt. 51, 8440-8448 (2012).

3. A. Giesen, H. Hugel, A. Voss, K. Wittig, U. Brauch, and H. Opower, "Scalable concept for diode-pumped high-power solid-state lasers,” Appl. Phys. B 58, 365-372 (1994).

4. Y. Ishida and K. Naganuma, "Compact diode-pumped all-solidstate femtosecond $\mathrm{Cr}^{4+}$ :YAG laser," Opt. Lett. 21, 51-53 (1996)

5. D. L. Sipes, "Highly efficient neodymium-yttrium aluminum garnet laser end pumped by a semiconductor-laser array," Appl. Phys. Lett. 47, 74-76 (1985).

6. P. Koopmann, S. Lamrini, K. Scholle, P. Fuhrberg, K. Petermann, and G. Huber, "Efficient diode-pumped laser operation of Tm: $\mathrm{Lu}_{2} \mathrm{O}_{3}$ around $2 \mu \mathrm{m}$," Opt. Lett. 36, 948-950 (2011).

7. D. Li, U. Demirbas, A. Benedick, A. Sennaroglu, J. G. Fujimoto, and F. X. Kärtner, "Attosecond timing jitter pulse trains from semiconductor saturable absorber mode-locked Cr:LiSAF lasers," Opt. Express 20, 23422-23435 (2012).

8. L. G. DeShazer and K. W. Kangas, "Extended infrared operation of titanium sapphire laser," in Conference on Lasers and Electro Optics (1987), Vol. 14, pp. 296-298.

9. R. Ell, U. Morgner, F. X. Kärtner, J. G. Fujimoto, E. P. Ippen, V. Scheuer, G. Angelow, and T. Tschudi, "Generation of 5 fs pulses and octave-spanning spectra directly from a Ti:sapphire laser," Opt. Lett. 26, 373-375 (2001).

10. P. F. Moulton, "Spectroscopic and laser characteristics of Ti: $\mathrm{Al}_{2} \mathrm{O}_{3}$," J. Opt. Soc. Am. B 3, 125-133 (1986).

11. P. W. Roth, A. J. Maclean, D. Burns, and A. J. Kemp, "Directly diode-laser-pumped Ti:sapphire laser," Opt. Lett. 34, 3334-3336 (2009).

12. M. D. Young, S. Backus, C. Durfee, and J. Squier, "Multiphoton imaging with a direct-diode pumped femtosecond Ti:sapphire laser," J. Microsc. 249, 83-86 (2013).

13. C. G. Durfee, T. Storz, J. Garlick, S. Hill, J. A. Squier, M. Kirchner, G. Taft, K. Shea, H. Kapteyn, M. Murnane, and S. Backus, "Direct diode-pumped Kerr-lens mode-locked Ti:sapphire laser," Opt. Express 20, 13677-13683 (2012).

14. P. W. Roth, A. J. Maclean, D. Burns, and A. J. Kemp, "Direct diode-laser pumping of a mode-locked Ti:sapphire laser," Opt. Lett. 36, 304-306 (2011).

15. P. W. Roth, D. Burns, and A. J. Kemp, "Power scaling of a directly diode-laser-pumped Ti:sapphire laser," Opt. Express 20, 20629-20634 (2012).

16. A. Müller, O. B. Jensen, A. Unterhuber, T. Le, A. Stingl, K.-H. Hasler, B. Sumpf, G. Erbert, P. E. Andersen, and P. M. Petersen, "Frequency-doubled DBR-tapered diode laser for direct pumping of Ti:sapphire lasers generating sub-20 fs pulses," Opt. Express 19, 12156-12163 (2011).

17. S. A. Payne, L. L. Chase, H. W. Newkirk, L. K. Smith, and W. F. Krupke, "LiCaAlF $6 \mathrm{Cr}^{3+}$ a promising new solid-state laser material,” IEEE J. Quantum Electron. 24, 2243-2252 (1988).

18. S. A. Payne, L. L. Chase, L. K. Smith, W. L. Kway, and H. W. Newkirk, "Laser performance of $\mathrm{LiSAIF}_{6}: \mathrm{Cr}^{3+}$," J. Appl. Phys. 66, 1051-1056 (1989). 
19. L. K. Smith, S. A. Payne, W. L. Kway, L. L. Chase, and B. H. T. Chai, "Investigation of the laser properties of $\mathrm{Cr}^{3+}: \mathrm{LiSrGaF}_{6}$," IEEE J. Quantum Electron. 28, 2612-2618 (1992).

20. Z. Chen and G. Zhang, "Free-running emerald laser pumped by laser diode," Chin. Opt. Lett. 4, 649-651 (2006).

21. S. T. Lai, "Highly efficient emerald laser," J. Opt. Soc. Am. B 4, 1286-1290 (1987)

22. J. C. Walling, "Tunable cw alexandrite lasers," J. Opt. Soc. Am. 69, 1436 (1979)

23. J. C. Walling, H. P. Jenssen, R. C. Morris, E. W. O'Dell, and O. G. Peterson, "Tunable laser performance in $\mathrm{BeAl}_{2} \mathrm{O}_{4} \mathrm{Cr}^{3+}$," Opt. Lett. 4, 182-183 (1979).

24. J. C. Walling, O. G. Peterson, H. P. Jenssen, R. C. Morris, and E. W. Odell, "Tunable alexandrite lasers," IEEE J. Quantum Electron. 16, 1302-1315 (1980)

25. J. C. Walling, D. F. Heller, H. Samelson, D. J. Harter, J. A. Pete, and R. C. Morris, "Tunable alexandrite lasers-development and performance,” IEEE J. Quantum Electron. 21, 1568-1581 (1985).

26. O. Svelto, Principles of Lasers (Plenum, 1989).

27. W. Denk, J. H. Strickler, and W. W. Webb, "Two-photon laser scanning fluorescence microscopy," Science 248, 73-76 (1990).

28. W. R. Zipfel, R. M. Williams, and W. W. Webb, "Nonlinear magic: multiphoton microscopy in the biosciences," Nat. Biotechnol. 21, 1369-1377 (2003).

29. C. Xu and W. W. Webb, "Measurement of two-photon excitation cross sections of molecular fluorophores with data from 690 to 1050 nm," J. Opt. Soc. Am. B 13, 481-491 (1996).

30. S. Sakadzic, U. Demirbas, T. R. Mempel, A. Moore, S. Ruvinskaya, D. A. Boas, A. Sennaroglu, F. X. Kartner, and J. G. Fujimoto, "Multi-photon microscopy with a low-cost and highly efficient Cr:LiCAF laser," Opt. Express 16, 20848-20863 (2008).

31. E. Sorokin, "Solid-state materials for few-cycle pulse generation and amplification," in Few-Cycle Laser Pulse Generation and Its Applications, F. X. Kärtner, ed., Vol. 95 of Topics in Applied Physics (Springer-Verlag, 2004), pp. 3-73.

32. U. Demirbas, D. Li, J. R. Birge, A. Sennaroglu, G. S. Petrich, L. A. Kolodziejski, F. X. Kärtner, and J. G. Fujimoto, "Low-cost, singlemode diode-pumped Cr:colquiriite lasers," Opt. Express 17, 14374-14388 (2009).

33. J. W. Kuper, T. Chin, and H. E. Aschoff, "Extended tuning of alexandrite laser at elevated temperatures," in Advanced Solid State Lasers, Vol. 6 of OSA Proceedings Series (Optical Society of America, 1990), paper CL3.

34. F. Druon, F. Balembois, and P. Georges, "New laser crystals for the generation of ultrashort pulses," C. R. Phys. 8, 153-164 (2007).

35. A. Sanchez, R. E. Fahey, A. J. Strauss, and R. L. Aggarwal, "Room-temperature continuous-wave operation of a $\mathrm{Ti}^{-\mathrm{Al}_{2} \mathrm{O}_{3}}$ laser," Opt. Lett. 11, 363-364 (1986).

36. R. Scheps, J. F. Myers, T. R. Glesne, and H. B. Serreze, "Monochromatic end-pumped operation of an alexandrite laser," Opt. Commun. 97, 363-366 (1993).

37. J. K. Jabczynski, W. Zendzian, Z. Mierczyk, and Z. Frukacz, "Chromium-doped LiCAF laser passively $Q$ switched with a $V^{3+}$ :YAG crystal," Appl. Opt. 40, 6638-6645 (2001).

38. M. L. Shand and J. C. Walling, "Excited-state absorption in the lasing wavelength region of alexandrite," IEEE J. Quantum Electron. 18, 1152-1155 (1982).

39. L. J. Atherton, S. A. Payne, and C. D. Brandle, "Oxide and fluoride laser crystals," Annu. Rev. Mater. Sci. 23, 453-502 (1993).

40. M. Stalder, M. Bass, and B. H. T. Chai, "Thermal quenching of fluoresence in chromium-doped fluoride laser crystals," J. Opt. Soc. Am. B 9, 2271-2273 (1992).

41. J. M. Eichenholz and M. Richardson, "Measurement of thermal lensing in $\mathrm{Cr}^{3+}$-doped colquiriites," IEEE J. Quantum Electron. 34, 910-919 (1998).

42. T. H. Maiman, "Stimulated optical radiation in ruby," Nature 187, 493-494 (1960).
43. W. Koechner, Solid-State Laser Engineering, 6th ed. (Springer, 2006).

44. M. Srotkamp, U. Witte, A. Munk, A. Hartung, S. Gausmann, S Hengesbach, M. Traub, J. Hoeffner, and B. Jungbluth, "Broadly tunable, diode pumped alexandrite laser," in Advanced SolidState Lasers (Optical Society of America, 2013), paper ATu3A.42.

45. J. C. Walling, O. G. Peterson, and R. C. Morris, "Tunable cw alexandrite laser," IEEE J. Quantum Electron. 16, 120-121 (1980).

46. H. Samelson and D. J. Harter, "High-pressure mercury arc lamp excited cw alexandrite lasers," in Conference on Lasers and Electro-Optics, Anaheim, California, June 19-22 1984 (Optical Society of America, 1984).

47. S. T. Lai and M. L. Shand, "High efficiency cw laser-pumped tunable alexandrite laser," J. Appl. Phys. 54, 56642-56644 (1984).

48. J. W. Kuper and D. C. Brown, "Green pumped alexandrite lasers," Proc. SPIE 5707, 265-270 (2005).

49. J. W. Kuper and D. C. Brown, "High efficiency CW green pumped alexandrite lasers," Proc. SPIE 6100, 61000T (2006).

50. M. Lando, Y. Shimony, R. M. J. Benmair, D. Abramovich, V. Krupkin, and A. Yogev, "Visible solar-pumped lasers," Opt. Mater. 13, 111-115 (1999).

51. R. Scheps, B. M. Gately, J. F. Myers, J. S. Krasinski, and D. F. Heller, "Alexandrite laser pumped by semiconductor-lasers," Appl. Phys. Lett. 56, 2288-2290 (1990).

52. X. Peng, A. Marrakchi, J. C. Walling, and D. F. Heller, "Watt-level red and UV output from a CW diode array-pumped tunable alexandrite laser," in Conference on Lasers and Electro-Optics (Optical Society of America, 2005), paper CMAA5.

53. M. J. Damzen, G. M. Thomas, and A. Minassian, "Multi-watt diode-pumped alexandrite laser operation," in CLEO Europe, Munich, May 12-16, 2013.

54. U. Demirbas, M. Schmalz, B. Sumpf, G. Erbert, G. S. Petrich, L. A. Kolodziejski, J. G. Fujimoto, F. X. Kärtner, and A. Leitenstorfer, "Femtosecond Cr:LiSAF and Cr:LiCAF lasers pumped by tapered diode lasers," Opt. Express 19, 20444-20461 (2011).

55. B. Sumpf, P. Adamiec, M. Zorn, H. Wenzel, and G. Erbert, "Nearly diffraction limited tapered lasers at $675 \mathrm{~nm}$ with $1 \mathrm{~W}$ output power and conversion efficiencies above 30\%," IEEE Photon. Technol. Lett. 23, 266-268 (2011).

56. E. Beyatll, A. Sennaroglu, and U. Demirbas, "Self- $Q$-switched Cr:LiCAF laser," J. Opt. Soc. Am. B 30, 914-921 (2013).

57. B. C. Weber and A. Hirth, "Efficient single-pulse emission with submicrosecond duration from a Cr:LiSAF laser," Opt. Commun. 128, 158-165 (1996)

58. B. C. Weber and A. Hirth, "Presentation of a new and simple technique of $Q$-switching with a $\operatorname{LiSrAlf}(6): \mathrm{Cr}^{3+}$ oscillator," Opt. Commun. 149, 301-306 (1998).

59. I. Freund, "Self- $Q$-switching in ruby lasers," Appl. Phys. Lett. 12, 388 (1968).

60. R. J. Collins, L. O. Braun, and D. R. Dean, "A new method of giant pulsing ruby lasers," Appl. Phys. Lett. 12, 392 (1968).

61. M. Birnbaum and C. L. Fincher, "The ruby laser: pumped by a pulsed argon ion laser," Appl. Phys. Lett. 12, 225-227 (1968).

62. A. Szabo and L. E. Erickson, "Self-Q-switching of ruby lasers at 77 degrees K," IEEE J. Quantum Electron. QE-4, 692 (1968).

63. D. Findlay and R. A. Clay, "The measurement of internal losses in 4-level lasers," Phys. Lett. 20, 277-278 (1966).

64. J. A. Caird, L. G. DeShazer, and J. Nella, "Characteristics of room-temperature $2.3-\mu \mathrm{m}$ laser emission from $\mathrm{Tm}^{3+}$ in YAG and $\mathrm{YAlO}_{3}$," IEEE J. Quantum Electron. 11, 874-881 (1975).

65. U. Demirbas, S. Eggert, and A. Leitenstorfer, "Compact and efficient Cr:LiSAF lasers pumped by one single-spatial-mode diode: a minimal cost approach,” J. Opt. Soc. Am. B 29, 1894-1903 (2012)

66. K. Torizuka, M. Yamashita, and T. Yabiku, "Continuous-wave alexandrite laser-pumped by a direct-current mercury arc lamp," Appl. Opt. 32, 7394-7398 (1993). 